\title{
Redesigning the diagnostic pathway for chest pain patients in emergency departments
}

\author{
Sebastian Rachuba ${ }^{1}$ - Andrew Salmon ${ }^{1} \cdot$ Zhivko Zhelev $^{1} \cdot$ Martin Pitt $^{1}$
}

Received: 30 June 2016 / Accepted: 9 March 2017 / Published online: 30 March 2017

(C) The Author(s) 2017. This article is published with open access at Springerlink.com

\begin{abstract}
Patients presenting with chest pain at an emergency department in the United Kingdom receive troponin tests to assess the likelihood of an acute myocardial infarction (AMI). Until recently, serial testing with two blood samples separated by at least six hours was necessary in order to analyse the change in troponin levels over time. New high-sensitivity troponin tests, however, allow the inter-test time to be shortened from six to three hours. Recent evidence also suggests that the new generation of troponin tests can be used to rule out AMI on the basis of a single test if patients at low risk of AMI present with very low cardiac troponin levels more than three hours after onset of worst pain. This paper presents a discrete event simulation model to assess the likely impact on the number of hospital admissions if emergency departments adopt strategies for serial and single testing based on the use of high-sensitivity troponin. Data sets from acute trusts in the South West of England are used to quantify the resulting benefits.
\end{abstract}

Keywords Emergency department - Diagnostic pathways - Chest pain - Avoidable admissions - Discrete event simulation $\cdot$ Case study

\section{Introduction}

Chest pain is among the most common reasons for attendance at emergency departments (EDs) in the United

Sebastian Rachuba

s.rachuba@exeter.ac.uk

1 University of Exeter, Medical School, St Luke's Campus, Exeter, EX1 2LU, UK
Kingdom (UK) and accounts for up to $6 \%$ of all ED visits per year [16]. This constitutes a population of roughly one million patients (with levels of attendance varying according to patient age, gender etc.). It therefore represents a significant workload for hospitals and their staff especially in the context of the trend of increasing ED attendances in recent years. Many of these ED attendances lead to a subsequent admission into a short stay ward, e.g. a Medical Assessment Unit (MAU), or into specialised (cardiac) wards. Such admissions are commonly triggered by the UK National Health Service (NHS) requirement that EDs avoid patients staying longer than four hours in the ED. This 4 hour target often leads to admissions into neighbouring wards, such as Clinical Decision Units (CDU), in order to avoid breaching the target. Some clinicians argue that, especially from a patient's perspective, it is beneficial not to be admitted, since this will reduce unnecessary tests and medications. Furthermore, whilst CDUs are commonly under the management of ED staff and transferring patients to CDUs does not reduce the workload for ED staff, admissions will have a greater adverse impact on a hospital's overall financial performance as well as that of the overall health system.

The likelihood of an AMI is usually gauged from an assessment of a patient's medical history, clinical examination, electro cardiograms (ECGs) and, in particular, serial cardiac troponin (cTn) tests. Cardiac troponin is a biomarker of myocardial necrosis and an increased concentration in the bloodstream indicates damage to the heart muscle. It is not, however, specific to AMI and various acute and chronic conditions could also lead to elevated cTn levels. In AMI, cTn shows a specific pattern of rapid increase which, together with evidence of myocardial ischemia, is used to diagnose AMI and initiate suitable treatment for the patient. In suspected AMI, cTn is measured in two blood samples taken 
several hours apart in order to distinguish between chronic conditions with stable increase in cTn concentration and AMI where rapid change in cTn levels is observed. If both samples are below a threshold, i.e. negative, and there is no evidence of rapid change, AMI can be ruled out. If no other concerns about the patient's medical condition are present, such patients can be discharged directly from the ED. If the change relative to the baseline troponin value, i.e. the first test's value, is significantly large, the patient is likely to have had a heart attack and special cardiac treatment is required immediately. Until recently, it was recommended that the first sample is taken at the patient's presentation to the ED and the second 10-12 hours after the onset of symptoms to allow enough time for the troponin values to rise and to be detected by the test [33]. The recently published guidance by the National Institute for Health and Care Excellence (NICE, [24]) suggests that with the high-sensitivity cTn assays the inter-test time could be made shorter to allow early rule-out of AMI in patients at low risk of the condition. According to these recommendations, in low risk patients, two blood samples - one at presentation and one three hours later - achieve sufficient accuracy to rule out AMI provided the patient does not present within three hours of symptom onset. Figure 1 explains troponin values as a function of time since onset of pain. It is also highlighted how recommendations by the NICE committee affect the practice of testing. Apart from serial testing, decisions can be based on a single test if the blood sample is taken at least six hours after the onset of worst pain.

The main focus of the research project was to investigate how the introduction of high-sensitivity cardiac troponin tests affected current practice at hospitals in the South West of England. Our study was conducted in collaboration with the South West Academic Health Science Network (SW AHSN) and acute trusts in the South West of England [2].
We worked in an interdisciplinary team with clinicians at the different hospitals, information specialists, public health and diagnostic research and operational researchers. Project results indicated significant improvements in reducing the number of hospital admissions [28]). Finally, recent studies report that some low risk patients could be safely discharged on the basis of a single negative test if they present more than three hours after the onset of worst symptoms and have very low levels of $\mathrm{cTn}[8,11,31,37]$. Our study aims to support EDs to effectively manage staff workload and to reduce the number of avoidable admissions from ED into hospital. The impact of pathway reconfigurations on these goals is addressed using suitable performance metrics identified in this paper and we discuss the following research questions:

1. What are the relevant performance metrics when looking at diagnostic pathways for chest pain patients?

2. How can evidence-based pathway changes be assessed using a discrete event simulation model?

3. How do the identified key performance indicators (KPIs) change if the inter-test interval is shortened to 3 hours?

4. How do strategies incorporating single test rule-out affect KPIs compared to current practice?

5. What are barriers and facilitators of a discrete event simulation study looking at a redesign of diagnostic pathways for chest pain?

In the following sections we provide necessary background information about the scope of this research project and discuss relevant literature in order to highlight the research gap this study aims to bridge (Section 3). This is then followed by a discussion of available data sets and suitable performance metrics in Section 4. The discrete event simulation model which will be presented in

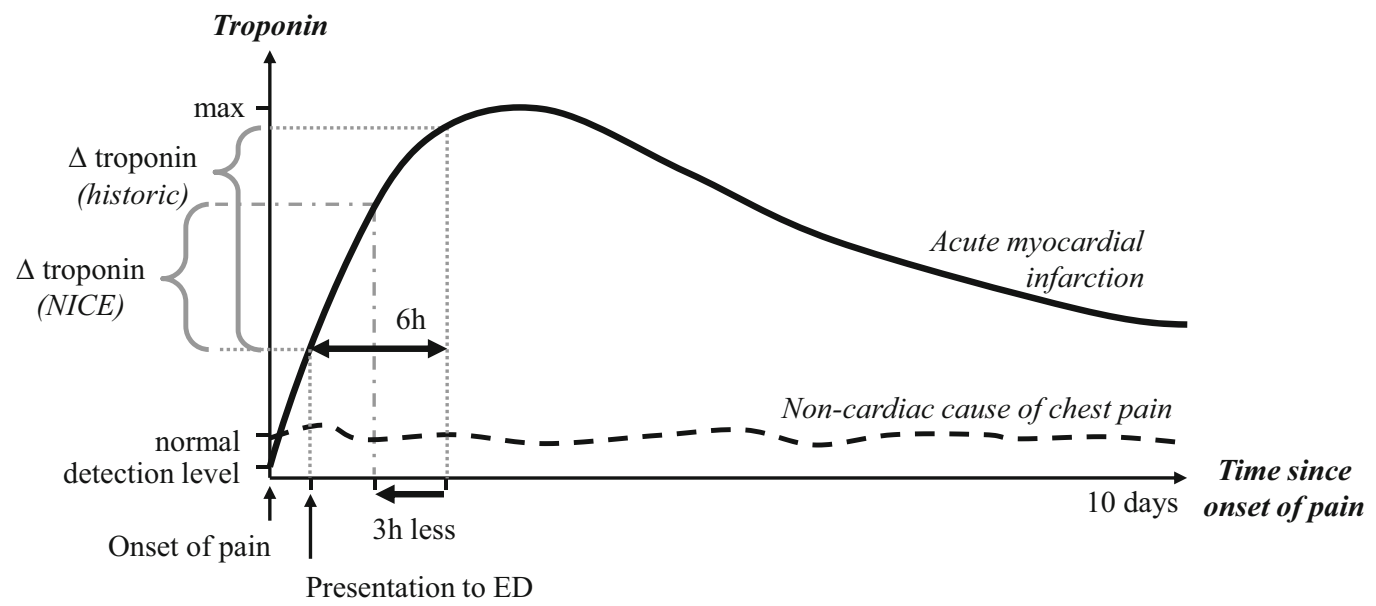

Fig. 1 Changes in troponin concentration over time for different medical conditions, i.e. acute myocardial infarction versus other causes of chest pain 
Section 5 merges project requirements and available data. We will finally use the model to conduct what-if analyses (Section 6). The last section discusses key findings of this study together with its limitations.

\section{Related literature}

A literature review for this project bears on two distinct areas of research which are jointly analysed. Firstly, diagnostic accuracy research and, more specifically, the accuracy of high-sensitivity cTn troponin tests, is a key input for our study. We analyse publications looking at pathway changes following the introduction of high-sensitivity cTn assays and their use with protocols for early rule-out of AMI. Secondly, discrete event simulation (DES) models have been widely used to model patient flow through emergency departments. This is narrowed down and the focus of this review is on studies in which the purpose of the models is to evaluate changes to existing pathways. Finally, we discuss this paper's contribution bringing together the two threads of literature and research.

\subsection{Diagnostic testing and accuracy of troponin tests}

Cardiac troponin tests are regarded as the gold standard for diagnosis of AMI. Previous versions of these assays, however, lacked the diagnostic sensitivity necessary for safe rule-out of AMI in the first few hours after patient presentation at the ED. As a result, a second measurement 10-12 hours from the onset of symptoms was recommended to avoid inadvertent discharge of patients with evolving AMI [33]. In the last five years, new sensitive and high-sensitivity assays have been developed, which are able to identify very small concentrations of cTn. These can reliably quantify levels below the 99th percentile of a healthy reference population used as a positivity cut-off for AMI. This makes it possible to identify increased levels of cTn early in the diagnostic process and to avoid long delays before AMI is finally ruled out.

In 2014, NICE issued guidelines recommending the use of high-sensitivity cTn assays for early rule-out of AMI [24]. Given the significant differences between the available cTn assays branded as sensitive or highly sensitive, NICE felt obliged to define a high-sensitivity cTn assay as those that meet the following two criteria: has a coefficient of variation of $10 \%$ or less at the 99th percentile (the upper limit of the reference population) and is able to detect cTn in at least $50 \%$ of the reference population. Only three assays met these criteria and only for two of them, the Elecsys Troponin T high-sensitive (Roche Diagnostics) and the ARCHITECT STAT High-Sensitive Troponin-I assay (Abbott Diagnostics), sufficient evidence was identified to recommend their use in clinical practice. The NICE guidance recommends that for low risk patients these assays could be used, in conjunction with all relevant clinical information, to rule out AMI early in the diagnostic process. Such protocols should be based on two samples taken at least three hours apart; the manufacturer-recommended cut-off based on the 99th percentile together with changes in cTn levels should be used as a decision threshold; and additional factors that might affect the performance of the test, such as the time elapsed from the onset of symptoms, the baseline cTn level and patients risk factors should be considered when making a decision. It was hoped that such early rule-out protocols could help avoid unnecessary waits and hospital admissions of patients who do not have AMI or other life-threatening conditions and that such patients could safely be discharged from the ED.

The recommendation to make a decision based on the change in cTn concentration between two blood samples reflects the need to distinguish between chronically elevated baseline troponin and the acute pattern of increase typical for AMI. Chronically elevated troponin could be observed in a range of medical conditions, such as pulmonary embolism, chronic heart failure, and coronary artery disease and also in patients with impaired renal function. Also, the baseline level of cTn is age-dependent and a significant proportion of older patients, especially those over 75 years of age, will have baseline cTn above the 99th percentile without having an AMI or another cardiac condition. For instance, [26] found that the median (inter-quartile range, IQR) value of Elecsys Troponin T high-sensitive in a reference population of presumably healthy individuals varied significantly across age groups. It was $6.33 \mathrm{ng} / \mathrm{L}$ (IQR: 4.1-9.4) in 50-75 years old, $28.6 \mathrm{ng} / \mathrm{L}$ (IQR: $23.5-37.9$ ) in 75-95 years old and $48.5 \mathrm{ng} / \mathrm{L}$ (IQR: $39.3-68.5$ ) in those $>96$ years old.

Although considering the change in cTn levels over time may help avoid misclassification of cases where cTn is elevated due to non-AMI reasons, in practice this leads to a significant delay in making a decision about admission or discharge. Given the expectation within NHS that at least 95\% of ED patients should be discharged or admitted to hospital within four hours of their presentation, implementing the above recommendation means that most of the patients will be admitted regardless of their level of risk and the result from the first cTn measurement. Once admitted, such patients will need to spend time in hospital until the result from the second blood sample is received and a consultant is available to make a decision.

Alternatives to this strategy have been proposed taking advantage of the fact that in patients with very low pre-test probability of AMI and Acute Coronary Syndrom (ACS), very low baseline cTn levels make the possibility of AMI highly unlikely $[8,11,31,37]$. If incorporated in the 
diagnostic pathway, such an early rule-out protocol could help avoid unnecessary tests and admissions for patients who do not have AMI but present with chest pain and have had cTn measured at presentation. Such a strategy does not obviate the need for a second cTn measurement which, for many patients, will still be required to rule out AMI. Also, such a strategy is not applicable to patients who present less than three hours after onset of symptoms because, as the studies referred to above suggest, the risk of AMI being missed in such patients is still considerable.

\subsection{Pathway redesign in emergency departments}

ED departments and their processes have been repeatedly modelled from a variety of perspectives. A recent overview in [17] outlines this variety in terms of resulting research questions. Many of the existing papers in this area study reasons for excessive patient waits and discuss strategies to overcome crowded emergency departments [25, 30, 34, 36]. Patient flows and overcrowding of departments appear to be common themes in recent years [12], both of which resonate with the purpose of our work.

DES is among the most commonly used techniques in healthcare operational research [22] and has been repeatedly deployed to address problems in ED [6], even to the extent that generic templates have been proposed [15], although these have not met with unanimous approval [12]. Common outcome measures of ED models appear to be the time spent in department, patient throughput and resource utilisation [6]. On the other hand, the costs of Emergency Departments appears to be an under researched topic [17]. The vast majority of models are very unit specific. Approaches taken include assessment of working practices [1, 3-5], shift patterns [5], equipment bottlenecks [9], and in some cases, patient pathways themselves [20]. Interactions of ED patient flow with the wider hospital context are clearly also important [7], and examination of these interactions can be achieved using DES models, or sometimes may be better analysed by other techniques [29, 32].

A recent trend has been the use of operational research models and methods, including DES, to address the flow of both sub-populations within ED, as well as aspects of specific treatment pathways, e.g. the time to treatment in acute stroke in which ED is closely involved [20]. Clearly, many EDs are resource limited and whereas purchase of equipment or extra staffing may not be an option [5], time and resource saving interventions that utilise existing staff may clearly be beneficial. With regards to high-sensitivity troponin, these topics have also been explored in clinical trials [31]. Whilst allowing for some assessment of impact, such investigations tend to be either expensive or time consuming, and do not allow the researcher to find the optimal combination of process changes in terms of resource and time benefits. Therefore, whilst there is evidence that high-sensitivity troponin testing (in particular single versus multiple point testing) is likely to be beneficial and safe, it is unclear how it should be implemented and what the actual benefits might be.

\subsection{Contribution of our study}

All of those issues outlined above are at the core of this modelling project and will feature in the concluding discussion. The main aim of our study is to bring together operational research and the management of diagnostic pathways. We therefore refer to the work of e.g. [31] and [20], yet we clearly use a different focus. In comparison to the study by [20], our approach focuses on a different patient subpopulation attending ED (patients with chest pain), considers national guidelines together with medical evidence and assesses effects on the number of admissions and discharges when a new diagnostic strategy is introduced. In particular, our study focuses on low risk patients presenting at ED and we investigate changes to a diagnostic pathway rather than to a treatment pathway. Our study can also be dissociated from clinical trials such as [31], as a discrete event simulation study provides a number of major advantages, i.e. time and cost savings, the ability to update the model as the parameters of the analysis change, and mitigation of the risks of introducing change directly. Furthermore, trials do not take account of the local context which might be different from hospital to hospital. Using local routine data makes it possible to consider local structures and processes and to explore how new strategies would work locally, allowing for the uncertainty in different scenarios.

To summarise, the simulation model presented here acts as a support tool for clinicians and decision makers at hospitals. It provides a means to quantify various changes to pathways in terms of the numbers of admissions and discharges, but still requires a clinician's judgement to determine which of those options should be chosen. The expert's opinion is key in this case, because some decision making factors cannot be appropriately quantified yet need to be taken into account.

\section{Diagnostic pathways for chest pain}

Capturing the current diagnostic pathways for chest pain in ED was a major step in the analysis of current practice at the collaborating trusts. Clinical protocols and discussions with clinicians during mapping sessions provided valuable input to develop a suitable pathway model. In this section, we firstly present a generic pathway as one of the key outcomes of these analyses and meetings. A set of relevant performance metrics is another key outcome which we 
discuss subsequently. Note, that these performance metrics can be represented through different data, subject to their availability at the hospitals. We revisit this in Section 5 after introducing the available data for this study (Section 4).

\subsection{Generic pathway}

A key task of this project was to explore the impact of high-sensitivity troponin tests on current practice at the hospitals. This was done by capturing the various diagnostic pathways on whiteboards (and later refined versions on paper and digital) during a series of meetings with interdisciplinary hospital teams. Visualising current practice in terms of patient-focused pathways helped to describe the sequence of necessary tasks which are performed if a patient with chest pain presents at the ED.

Although individual protocols at the hospitals differed, a common pathway could be developed which is informed through the various meetings held across hospitals in the South West of England. The explicit focus of this project was on the management of patients at low risk of AMI. Patients presenting with a high-risk features, such as ongoing anginal chest pain or ST-segment elevation on the ECG, will be admitted regardless of the first troponin result and will not follow the clinical pathway described below [23, 24]. Figure 2 represents the generic steps of such a pathway. The dashed boxes at the beginning of the process highlight the two main ways of arriving at the hospital: Patients arrive by either ambulance or any other means of transportation which we refer to as self-presenting since they do not require medical assistance during the transport. Selfpresenting patients register their attendance at reception before they are triaged. Ambulance patients usually bypass registration and go straight to triage. The first blood sample is taken during the first medical assessment which is nurseled at some of the trusts. Once the blood has been drawn, the sample is dispatched to the laboratory to be analysed and, finally, results are reported electronically and made available to clinicians via the hospital's information system. After this first assessment, patients usually wait in an ED waiting room to be seen by a senior doctor or consultant. A decision on further management and treatments for the patient is made during the second assessment. Depending on the time interval between onset of pain and first sample taken at hospital, either a serial or a single test strategy can be performed. If the first blood sample is taken at least six hours after the onset of worst pain and the outcome is negative a patient can be discharged safely, if the doctor has no other concerns (single test strategy). According to the patient's overall medical condition, the discharge could require a follow-up meeting with a GP. A positive first sample taken more than six hours from onset of pain will lead to an admission into specialised care. A second test is usually required if the first sample is taken earlier than six hours from onset of pain regardless of the outcome of the first test (serial test strategy). NICE [24] currently does not recommend decisions based on a single test, even if negative. For this second test, patients are usually admitted to either CDU or MAU, where blood is drawn again and a sample is sent to the laboratory for analysis. Once available via the hospital's information system, the results for the two samples are jointly analysed and a decision is made whether to admit or discharge the patient. On rare occasions patients need to be admitted into cardiac care or other areas of specialised care, those are summarised under Admit: ward (inpatients). A very small number of samples (not more than 3\%) need to be repeated because the initial sample is haemolysed and cannot be used for analysis.

This generic description of the diagnostic pathway reflects the practice of most of the acute trusts in our study. However, there are some minor differences which are not relevant to this study. Some of the trusts do have short stay wards under the management of ED, some only have an MAU which is generally not under management of ED staff. There are also distinctions in terms of responsibilities for taking blood samples. Some hospitals require this to be done by a doctor (sometimes during a see and treat assessment), whilst others allow this to be performed by suitably qualified nurses. These disparities mean that it would be challenging to transfer examples of good practice between hospitals in our study, an important implication for the implementation of any proposed changes at specific EDs (however these aspects were outside the scope of our study). The case study presented below focuses on one of the

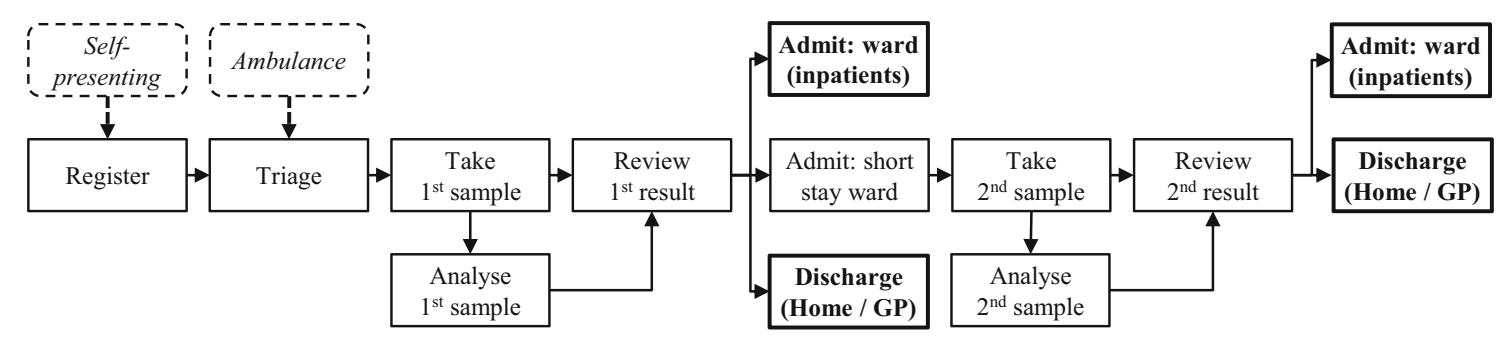

Fig. 2 Generic patient pathway in ED for patients presenting with chest pain at low risk of AMI 
collaborating trusts for which the generic pathway accurately represents the hospital's actual ED pathway.

\subsection{Performance metrics}

Discussions with clinicians identified the following two main performance metrics which are closely linked; the number of admissions and the resulting workload for ED staff. The number of admissions into hospital not only impacts a hospital's financial performance but it mainly causes additional workload. Also, the length of stay in the department is relevant to any patient attending ED. We will briefly discuss why these metrics are important to assess the performance of an ED.

\subsubsection{Number of admissions}

Admissions following the ED attendance caused by a suspected heart attack occurs with the majority of patients. For hospitals this increases their payments even if the patient is 'only' admitted to a short stay ward. From a different perspective, this leads to higher tariffs being paid and thus higher costs for the commissioner. As chest pain is among the most common reasons for ED attendance (approximately $2.4 \%$ to $6 \%$ per year [16]), medium-sized trusts have to deal with 8,000 patients or more every year. For the vast majority of patients, an admission into hospital would not have been necessary as they left the hospital without admission into specialised care. Hence, reducing the number of those admissions would reduce workload particularly for short stay wards.

\subsubsection{Number of single test decisions}

The above criterion is closely linked to the number of decisions based on a single test. Current practice is that a decision is made on only one sample if the time interval between the onset of pain and the first blood test is sufficiently large. According to studies investigating prehospital delays of patients, the probability of patients having blood taken less than six hours from onset of pain is very high. With the introduction of single test rule-out strategies based on high-sensitivity troponin, this time limit could be lowered from six hours down to four hours or less. This reduction will increase the number of decisions based on a single test and also positively influence the number of patients cared for by ED staff on short stay wards such as CDUs.

\subsubsection{Length of stay}

Apart from patient volume data, the length of stay in the emergency department is another key metric. In England, the Department of Health introduced the 4 hour target for EDs in 2004. This guideline demands that $95 \%$ of all attendances should have spent not more than four hours in ED [14]. In addition, the combined length of stay in both ED and on a short stay ward, particularly the CDU, is of similar importance. Reductions in either time interval mean that patients require less attention or observation from ED staff whilst in ED or CDU respectively.

Our simulation study investigates two potential changes: (1) reducing the inter-test time only and (2) reducing the inter-test time together with the limit for a single test strategy, i.e. the minimum required time between onset of pain and the first blood sample taken at ED. Both these options allow us to assess the number of admissions and hence quantify the beneficial effects of highsensitivity troponin tests. Clearly, a shorter time between two blood samples reduces a patient's overall length of stay in hospital and particularly the time spent on a short stay ward. In this case, the number of admissions might not necessarily change, yet the time during which a patient needs to be cared for on short stay wards could be reduced.

\section{Data analysis}

Historic data sets from the collaborating trusts were analysed in order to better understand the current use of troponin tests across the South West of England. The available data records were then employed to populate the DES model. Most hospitals provided background data for the patient cohort such as age, gender or mode of arrival. The available time stamps allowed us to investigate typical key process times, e.g. arrival to triage or arrival to sample. Clinical information, such as presenting complaint (i.e. the symptoms a patient describes during registration), working diagnosis (usually determined by a clinician or nurse during assessment), number of troponin tests and numerical test outcomes, were also provided. Either presenting complaint or working diagnosis was used as a filter criterion by hospitals' information departments to identify the appropriate population of interest. Medical doctors within our research team then checked free text fields - where available - describing the presenting complaint for consistency, i.e. whether the complaint recorded matched the diagnosis one would expect with acute coronary syndrome. The analyses of the data revealed very similar structures across the different trusts. Therefore, the presented figures can be considered representative for acute hospitals in the South West of England. There are differences in terms of volume data and the resulting absolute figures, but ratios relative to the number of patients considered are at very similar levels. 


\subsection{Patient cohort}

For the case study presented in this paper we use data from one of the hospitals (Trust A). A total number of over 8,200 patient episodes over a period of 12 months were included in the analysis. This marks the largest data set obtained for this study. Out of seven participating trusts, five provided data over a period of at least 12 months ranging between 1,200 and 8,200 patient episodes. This indicates different sizes of catchment areas for the collaborating hospitals. The share of male patients attending ED was higher $(56 \%)$ than female (44\%) patients for this trust. The share of male patients ranged from 55\% to 59\% across the trusts. Male patients also were on average younger (60.1 yrs) than female (61.6 yrs) patients. The overall population average for Trust A was 60.8 years with a median of 64 . Again, this is in line with data across the trusts, where male patients were on average between 56 and 63 years old and between two to four years younger than female patients. In general, the share of women increased in higher age categories, especially among attendees older than 75 years. The likelihood of requiring medical assistance to get to the hospital, i.e. travelling via ambulance, significantly increased with the patients' age. Across all trusts, between 69 and $76 \%$ of all arrivals were ambulance arrivals. One might expect this share to be higher in rural areas due to limited easy access to other means of transportation such as public transport but we did not identify such structural differences.

From observation there appears to be no seasonal pattern in the attendance, but a difference in weekdays and weekends is apparent. The most important feature is a typical intra-day variation in arrivals; all these effects are shown in Fig. 3. The level of attendance typically reached its maximum around noon and its minimum during night hours and very early mornings. Although attendance decreased from noon until midnight there were minor peaks, usually around $6 \mathrm{pm}$. This pattern demonstrates that most patients with chest pain attend an emergency department during morning times (possibly before work) or after work as the

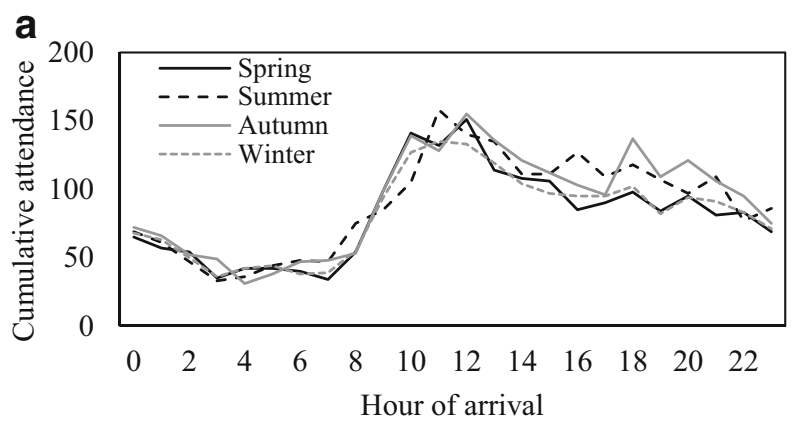

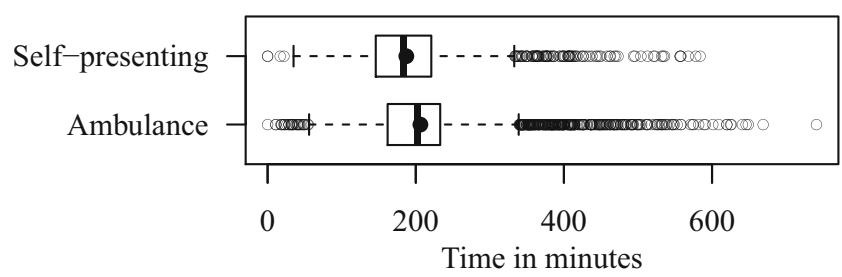

Fig. 4 Compared length of stay in ED for patients arriving by ambulance and self-presenting compared. Dots within the boxes represent the mean length of stay for ambulance arrivals $(206.3 \mathrm{~min})$ and self-presenting arrivals (187.3 $\mathrm{min}$ ) respectively

afternoon peak suggests. This pattern becomes less notable with increasing age because older patients are more likely to have a serious cardiac condition and thus attend the ED whenever necessary. A distinction between ambulance arrivals and self-presenting patients showed a pattern very similar to those mentioned above with ambulances arrivals proportionately higher due to higher volume in attendance.

\subsection{Process times}

The collaborating trusts provided various time stamps which mark the start of the pathway related activities (see Fig. 2). This allowed us to analyse start-to-start relations of activities and measure relevant process times. Most of the process times were linked to the arrival pattern and thus the resulting workload, i.e. processes tended to take longer during busy periods and length of stay in ED was longer for patients who arrived by ambulance since ambulance arrivals very often presented with a more severe condition. The boxplots in Fig. 4 highlight slightly longer length of stays for ambulance arrivals - both on average and in general as indicated by the shifted inter-quartile limits. Patients who were subsequently admitted to hospital tended to stay longer in ED. The level of attendance is naturally linked to the inter-arrival times. Figure 5 depicts the time between two arrivals according to means of transportation. Higher variation can be observed here with non-ambulance arrivals. This underlines that more

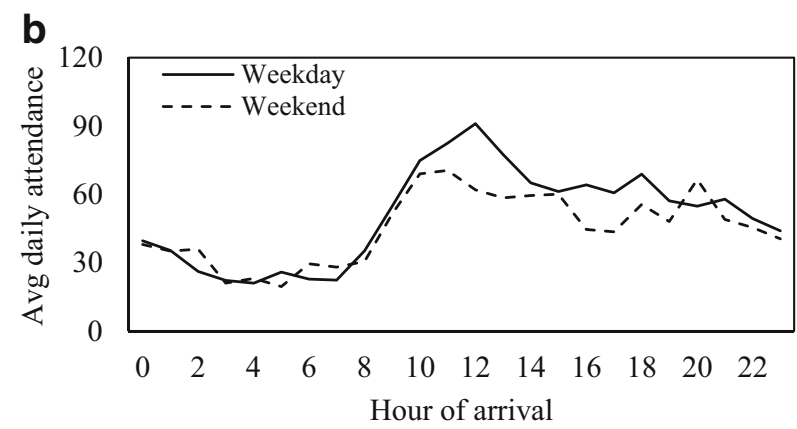

Fig. 3 Attendance of patients with chest pain at Trust A over a period of 12 months. The two graphs outline attendance (a) per season (cumulative) and (b) on weekdays versus weekends (daily average) 


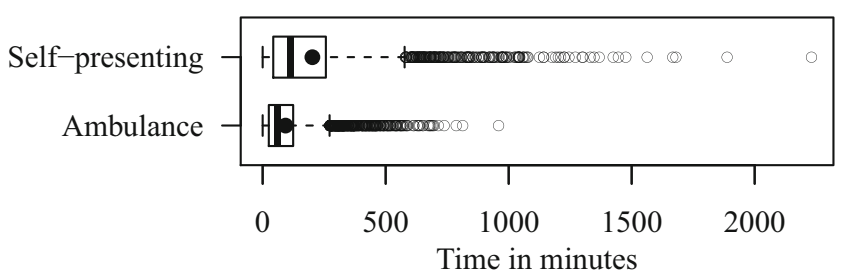

Fig. 5 Inter-arrival times for patients presenting with chest pain at ED. On average, self-presenting patients arrived every $202.1 \mathrm{~min}$ and ambulance arrivals occurred every $93.1 \mathrm{~min}$ (indicated by dots within the boxes)

serious causes of chest pain are linked to ambulance arrivals and these patients decide to attend the ED when they experience pain rather than delaying their attendance, e.g. until after they finish work. The time from arrival to triage is only relevant for non-ambulance arrivals, because for ambulance arrivals, delays before triage could only occur while the ambulance crew hands over a patient to clinical staff. The latter, however, would not affect the length of stay in ED because the attendance technically starts with the triage after the patient was handed over. For non-ambulance arrivals, the average time from arrival to triage was $23 \mathrm{~min}$ (SD $28 \mathrm{~min}$ ). Waits before triage were shorter during less busy times of the day (mean $18 \mathrm{~min}, \mathrm{SD} 22 \mathrm{~min}$ ) and longer during peak times respectively (mean $30 \mathrm{~min}$, SD $35 \mathrm{~min}$ ).

The time between arrival at ED and when the first blood sample taken is crucial to inform a doctor's decision whether a patient is eligible for either a single or a serial test strategy. The intra-day variation within the time interval between arrival at ED and the first blood sample taken varies over the day (see Fig. 6). During less frequented times of the day, i.e. during early morning hours, the difference between the overall daily average (dashed line) is significant. With rising numbers of arrivals towards noon and afternoon, the hourly average time until a first blood sample is taken is close to and above the plotted overall daily average (mean $55.7 \mathrm{~min}$, SD $42.4 \mathrm{~min}$ ). The total turnaround times for blood samples, i.e. the time including transportation and analysis until results are finally made available via the hospital's information system, are on average $91.2 \mathrm{~min}$ (SD $86.3 \mathrm{~min}$ ). In less than $3 \%$ of all cases, a blood sample cannot be used when arrived at the laboratory, i.e. it is haemolysed. This requires a new blood sample to be taken, transported and analysed. Those rare cases are not considered in the average time previously mentioned but are incorporated within the discrete event simulation model.

\subsection{Impact of the 4 hour target in ED on test strategies}

The strategy of serial troponin testing will almost always lead to admissions into CDU or MAU where the second blood sample is drawn and a final decision about admission or discharge is made. Through these admissions, breaches to the 4 hour target are avoided since it is likely that, after the second blood sample is taken, the timespan of arrival to first sample plus the inter-sample time add up to at least 240 min on average (see Fig. 7). Short stay wards attached to an emergency department, such as CDUs, are usually under control of ED staff. Hence, a reduction of the overall length of stay through an earlier discharge from short stay wards is likely to reduce workload for clinicians, nurses and administrative staff working in ED as well as removing the involvement of other departments and staff at the trust (i.e. it simplifies the patient pathway and reduces the time during which a patient receives care).

\subsection{Assumptions due to unavailability of data}

Not all information needed to populate the simulation model could be derived from existing data records. The time between onset of worst pain and drawing the first blood sample is crucial, because it triggers whether a single or serial test strategy needs to be performed. Unfortunately, this interval is only partially available through routinely collected data. We therefore separated out the time from onset of worst pain to arrival at hospital (pre-hospital delay) and the hospital process arrival to first sample in order to provide suitable replacements for the pain to sample time as shown in Fig. 8. However, since most of the trusts did not routinely record the time from onset of pain to arrival at the hospital, we made use of available studies from academic literature. Out of numerous studies on pre-hospital delays $[10,18,19,21,27]$ only few were suitable for this comparison because they took into account conditions similar to those in our study. In particular, we focused on two
Fig. 6 Timespan arrival to sample by hour of day. The dashed horizontal line represents the overall daily average

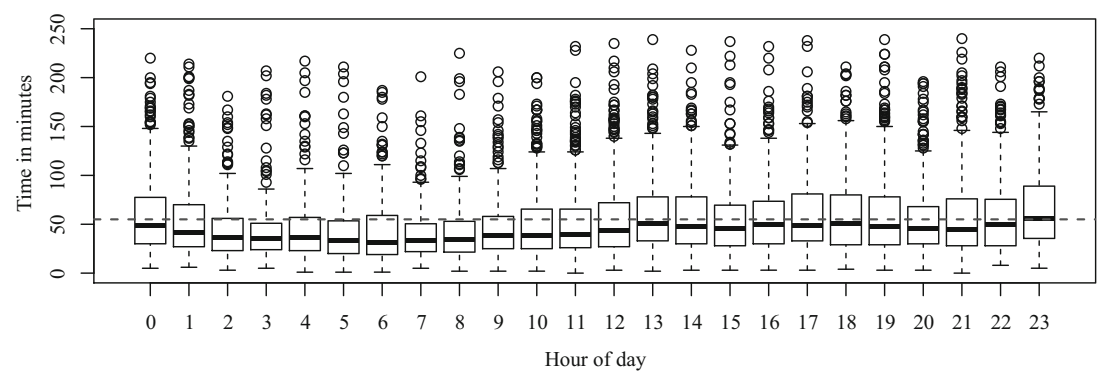


Fig. 7 Simplified pathway explaining the likelihood of breaching the 4 hour target when using a serial test strategy

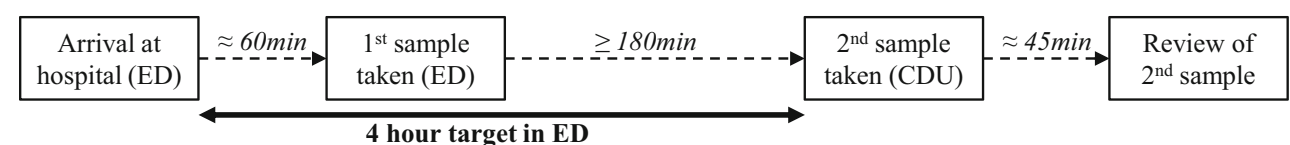

4 hour target in ED publications which report a study conducted in the UK and one focusing on the likely differences in delays when looking at onset of first and worst pain. The probability distributions in Fig. 9 follow a very similar pattern and there is only a little difference between the UK study in [27] and the worst pain to sample interval reported in [19]. In all studies the reported data follows a log-normal distribution with different parameters with a mean varying between $210 \mathrm{~min}$ and $360 \mathrm{~min}$ - the median is roughly $140 \mathrm{~min}$ ( $\pm 15 \mathrm{~min}$ ). However, the standard deviation varies significantly between 290 $\min$ and $560 \mathrm{~min}$. The distribution we used as a start in this study had a mean of $360 \mathrm{~min}$ and a standard deviation of roughly $240 \mathrm{~min}$. We found that using published data for our setting overestimated the number of patients who arrived in time to be managed on the basis of a single test only. By reducing the modelled delay in increments of $30 \mathrm{~min}$ utes we found that a mean pre-hospital delay of $300 \mathrm{~min}$ (together with an adjusted standard deviation of $250 \mathrm{~min}$ ) gave the best agreement with the trust data in terms of the number of patients receiving single or serial test rule out. This likely accounts for the apparent differences in travel times compared to the pre-hospital delays reported in [27]. Figure 9 shows how the chosen distribution for our model differs from other studies.

\subsection{Modelling of acuity levels of patients}

Together with clinicians and diagnostic experts, we developed four major categories of patients which can be interpreted as umbrella terms for the summarised diagnoses. We distinguished (1) high-risk and low-risk patients, the latter being further explained through (2) cardiac, (3) musculoskeletal and (4) other reasons of chest pain. In our simulation model, based on the data provided from Trust A, only $1.7 \%$ of patients are at high risk. The remaining groups account for $31.1 \%$ (cardiac causes), $28.6 \%$ (musculoskeletal causes), $38.6 \%$ (other causes) of the attending population respectively. Published research papers revealed two major patterns which troponin values could follow over time as previously shown in Fig. 1. Patients with AMI have a fast increase in their troponin concentration shortly after the onset of pain $[10,35]$, whereas with other medical conditions, e.g. musculoskeletal causes of chest pain, it is common that troponin concentration varies around a normal level. According to the patients' acuity levels, distinct troponin profiles over time were modelled to incorporate (1) a rapid increase in the concentration for high-risk patients and (2) a natural variation around a baseline concentration (see appendix). Performing serial testing captures the change in troponin values over time. A significant increase in the level of troponin would be an indicator for AMI. Conversely, only small changes in the level of this biomarker indicate that the heart muscle is not severely damaged and can be considered due to natural variation or a chronic condition. Upon arrival of patients in our model, we assigned the acuity level to patient-specific label which e.g. helped to route patients to cardiac wards and other specialised care.

\subsection{Making use of historic data}

We used basic maximum-likelihood fitting provided through dedicated $\mathrm{R}$ packages such as fitdistrplus or rriskDistributions to estimate key parameters for the distributions. All process times within the ED and the laboratory could be accurately described using gamma distributions. The most appropriate way to identify suitable distributions was to inspect the shape of histograms before estimating parameters for the respective candidate distributions. We focused on commonly used exponential, gamma and log-normal distributions as suitable candidates. We then inspected historical against theoretical quantiles for eligible distributions using quantile-quantile plots (QQ plots). This was necessary since the quality of statistical methods usually employed to test for a certain distribution is very low with large sample sizes. The inter-arrival times for both modes of arrival could also best be modelled using a gamma distribution. Again, this was accurately visualised using QQ plots. We decided to separate out the inter-arrival and process times in intervals of four hours starting at midnight. This is in line with recent publications in healthcare and
Fig. 8 Process times from onset of pain to (a) arrival and (a) blood drawn explained together with available proxies

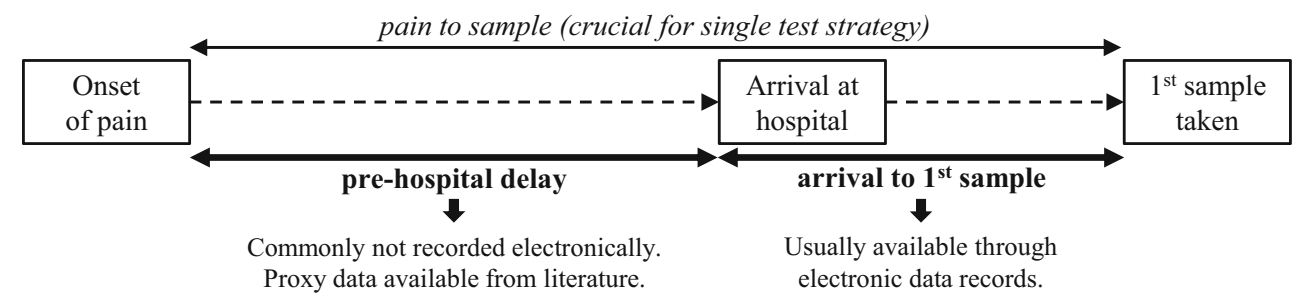




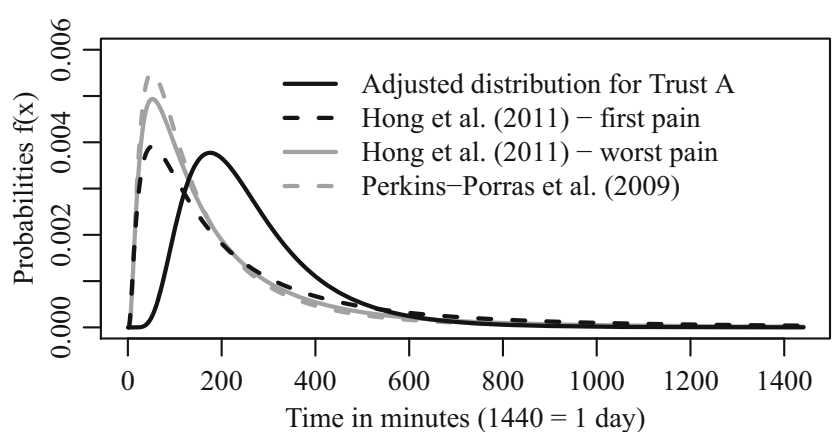

Fig. 9 Adjusted distribution modelling the pre-hospital delay compared to distributions reported in suitable studies in the literature

leads to sufficient level of detail [13]. The pre-hospital delay was best described in terms of a log-normal distribution.

\section{Discrete event simulation model}

We modelled this diagnostic pathway for patients with chest pain using Simul8 (Simul8 Corporation). The DES model depicted in Fig. 10 is based on the generic pathway outlined in Fig. 2. The underlying structure evolved over a number of pathway mapping sessions with multidisciplinary teams at hospitals across the South West of England. During those meetings the current practices at the hospitals were captured which also allowed to explore how close the current practice was to the respective clinical protocols.

\subsection{Model logic}

Patients arrive with inter-arrival times sampled from a timedepended gamma distribution which is separately defined for ambulance and non-ambulance arrivals. Each patient is assigned a diagnostic category, i.e. high-risk or low-risk with different causes of chest pain. Only non-ambulance arrivals register, before all patients are triaged and a first blood sample is drawn. Here, the work item is duplicated to individually simulate the process of analysing the blood sample. The time between patient arrival and first blood

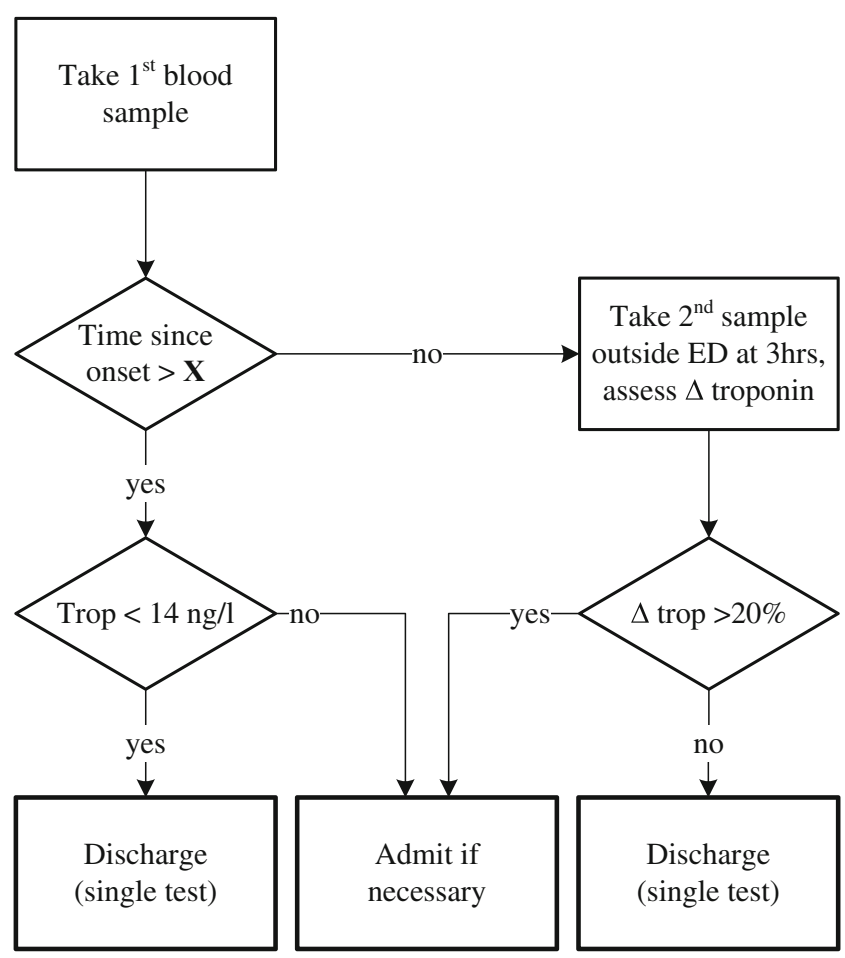

Fig. 11 Simplified decision logic within the DES model for low risk patients to determine whether single or serial strategy is applied

sample taken together with the pre-hospital delay records a patient's first troponin value once the sample has arrived at the lab. While this takes place, the patient in ED moves on to be seen by the doctor and, if troponin test results are available, a decision is made. In our model, medical decision making uses two characteristics, namely a patient's medical condition and the resulting level of troponin as the key factors. Those factors drive further management of the patient (i.e. whether it is appropriate to discharge the patient or whether an admission together with a second sample or even further treatment is required).

Figure 11 highlights the underlying decision principle which simplifies the original clinical protocol and has been discussed with, and approved by, collaborating clinicians. If the sample is taken more than six hours from onset of

Fig. 10 Screen capture of the DES model developed for Trust A using the software package Simul8. The blocks (arrivals, ED, laboratory, hospital admissions and discharges) visualise key elements of the diagnostic pathway

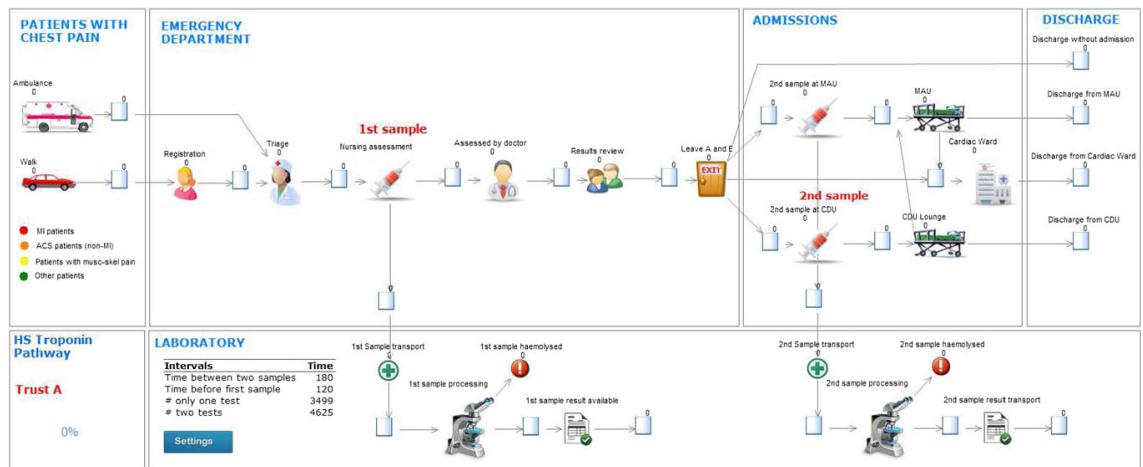


Table 1 Model validation comparing key parameters of the simulation model with historic data and deviation relative to historic values

\begin{tabular}{|c|c|c|c|}
\hline Parameter & Historic & DES model & Deviation \\
\hline \multicolumn{4}{|l|}{ Attendance } \\
\hline Ambulance & 5659 & 5637 & $0.40 \%$ \\
\hline Self-Presenting & 2580 & 2662 & $3.20 \%$ \\
\hline$L O S$ in $E D$ (in min) & 200.33 & 201.69 & $0.70 \%$ \\
\hline \multicolumn{4}{|l|}{ Outcome } \\
\hline Discharges (single test) & 1660 & 1749 & $5.40 \%$ \\
\hline Admission CDU & 1815 & 1758 & $3.10 \%$ \\
\hline Admissions MAU & 4015 & 4039 & $0.60 \%$ \\
\hline Other admissions & 749 & 745 & $0.50 \%$ \\
\hline
\end{tabular}

pain a decision on whether to admit or discharge a patient is made based on the troponin level and the overall medical condition of the patient, i.e. patients at low risk with a negative test and no other symptoms could be discharged. Patients presenting with high risk (i.e. clear symptoms of a heart attack) will be admitted regardless of the outcome of the troponin test. Those admissions are mainly based on assessing the patient's medical condition, the medical history, and other tests, in particular ECG. All patients with time since onset less than X hours (this is the hospital's choice) are then admitted to either MAU (positive first test) or CDU (negative first test) where a second blood sample is taken at least three hours after the first one. At this stage, the work item is again duplicated to capture the blood sample being sent to the laboratory for analysis. Once those second test results are available, a decision is made and patients are either admitted (given the change in troponin is significantly large) or discharged.
Initially, a generic prototype model was developed to represent the similarities in the diagnostic pathways for patients with chest pain across the participating trusts. This prototype model was subsequently tailored around practice at the acute hospitals. For instance, at Trust A it was common to have a review of troponin test results separate from the consultation, i.e. the consultation took place regardless of results being available. A separate consultation then assessed the results once they became available. Decision makers and clinicians can use a dedicated interface to change model parameters such as threshold for single test strategies and the inter-test time for serial testing.

\subsection{Model validation}

The validation of our model was done as a comparison of key model parameters and performance indicators against historic data. We performed a trial with 100 independent runs over a whole year and the results are shown in Table 1. For all relevant performance criteria, i.e. length of stay and the numbers of admissions and discharges. A sufficiently accurate representation of the current situation could be observed. The model slightly underestimates the number of admissions into CDU and, conversely, slightly overestimates the number of discharges based on a single test. This might be due to the troponin variation over time being modelled as a moving average process.

\section{Simulation study}

In order to investigate how both NICE guidelines and additional clinical evidence affect key performance indicators we specified a number of what-if scenarios. We compared the current system to those scenarios and quantified changes

Table 2 Absolute changes in length of stay in ED only and ED and CDU when complying with NICE guidelines and clinical evidence

\begin{tabular}{|c|c|c|c|c|c|}
\hline \multirow[t]{2}{*}{ Scenarios } & \multirow{2}{*}{$\begin{array}{l}\text { Limit for single testing /inter- } \\
\text { sample time (in min) }\end{array}$} & \multicolumn{2}{|c|}{ Time in ED \& CDU (in min) } & \multicolumn{2}{|c|}{ Time in ED only (in min) } \\
\hline & & Average & 95\% Conf. Int. & Average & $95 \%$ Conf. Int. \\
\hline Status Quo & $360 / 360$ & 546.24 & {$[545.99,546.48]$} & 201.69 & {$[201.51,201.87]$} \\
\hline NICE & $360 / 180$ & 366.24 & {$[365.99,366.48]$} & 201.69 & {$[201.51,201.87]$} \\
\hline S1 & $330 / 180$ & 361.18 & {$[360.90,361.45]$} & 198.45 & {$[198.27,198.63]$} \\
\hline S2 & $300 / 180$ & 355.66 & {$[355.32,355.99]$} & 195.98 & {$[195.81,196.16]$} \\
\hline S3 & $270 / 180$ & 348.70 & {$[348.35,349.05]$} & 193.89 & {$[193.71,194.07]$} \\
\hline S4 & $240 / 180$ & 347.84 & {$[347.49,348.19]$} & 193.62 & {$[193.44,193.80]$} \\
\hline S5 & $210 / 180$ & 343.25 & {$[342.92,343.58]$} & 192.23 & {$[192.05,192.41]$} \\
\hline S6 & $180 / 180$ & 335.22 & {$[334.79,335.65]$} & 190.38 & {$[190.20,190.56]$} \\
\hline S7 & $150 / 180$ & 322.06 & {$[321.52,322.60]$} & 189.30 & {$[190.20,189.48]$} \\
\hline S8 & $120 / 180$ & 319.98 & {$[319.39,320.57]$} & 189.15 & {$[188.96,189.33]$} \\
\hline
\end{tabular}


in length of stay in ED, length of stay in ED and CDU combined and finally the numbers of discharges versus admissions into CDU.

\subsection{Study design}

In total, nine different what-if scenarios were studied in comparison to the current system (see also Table 2). Scenario NICE modelled the recommendations made in the NICE guidelines, i.e. reducing the inter-test time from six to three hours. As shown in the Table 2, all other scenarios incorporate the newly suggested inter-sample time of 180 minutes. Scenarios S1 to S8 shorten the time limits for single test strategies in steps of 30 minutes. We conducted trials in which we simulated a whole year over 100 independent runs. No warm-up period was specified as this was not required given the dynamics of the model. The confidence intervals around the simulated mean were sufficiently small across the performance indicators. The CDU at Trust A can usually take up to 20 patients and is equipped with beds and chairs. At the same time, no more than 40 patients can be admitted into MAU. The MAU is occasionally sub-divided to assign beds to medical specialities when required.

\subsection{Results}

As previously discussed, the reduction of inter-test time did not affect the number of admissions at all as the second sample was always taken after a patient had been admitted (typically into CDU) regardless of the time between blood samples. Thus, only the time spent in ED and CDU was affected by adapting to national guidelines. A general overview of the impact on length of stay in ED only and in ED and CDU combined is shown in Table 2. Lowering the limit for single test rule-out strategies shortened the length of stay in ED and also the overall length of stay in ED and CDU. The shorter stays (which include short stay wards) resulted in less busy departments because the number of CDU admissions also decreased significantly with a lower time cut-off for single test strategies. Shorter times only in ED were closely linked to the increase in the number of discharges directly from ED. Generally, patients spent less time between having their blood test reviewed and leaving the department when they were subsequently discharged. This means that with more discharges directly from ED (and thus fewer admissions) the time in ED could be reduced by up to $12 \mathrm{~min}$ on average. A bigger reduction of $41 \mathrm{~min}$ resulted at the same time for patients being admitted into the CDU. Figure 12 supports those benefits and particularly highlights relative changes where $100 \%$ indicates compliance with NICE guidelines and an interval of at least 6 hours between onset of pain and the blood sample taken (single

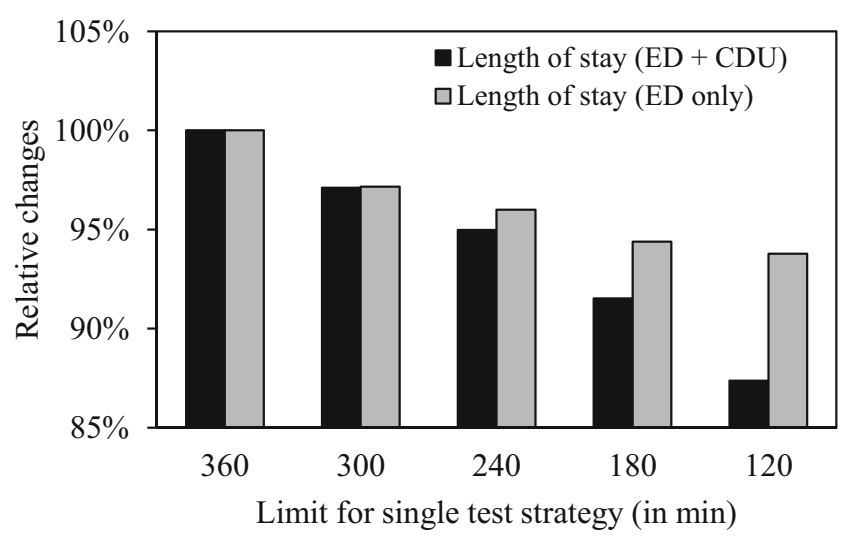

Fig. 12 Relative changes of LOS in ED only and LOS in ED and CDU respectively - the current setting marks the $100 \%$ level

test strategy). In terms of relative changes, the patients still being admitted for a second sample would benefit significantly. The most significant benefits occur in terms of the number of admissions into CDU and discharges from ED without admission respectively. Single test strategies mainly impact the numbers of discharges based on a single test, i.e. straight from ED, and the related number of admissions to CDU. Figure 13 outlines a shift from predominantly short stay admissions and only few discharges towards more discharges. It is worth mentioning that admissions into MAU were not affected in the what-if analyses. This is the case because patients admitted into MAU would had a positive first test, i.e. a troponin concentration of at least $14 \mathrm{ng} / \mathrm{litre}$, and thus, the number of MAU admissions did not change with a decreasing time cut-off. Figure 14 shows relative effects for CDU admissions and discharges based on a single test. Admissions to CDU could almost be eliminated, when allowing a 2 hour delay between the onset of pain and the time the blood sample was taken. All of those patients could potentially be discharged on the basis of a single test.

The described effects will likely be less strong in a setting where the average delay between onset of pain and

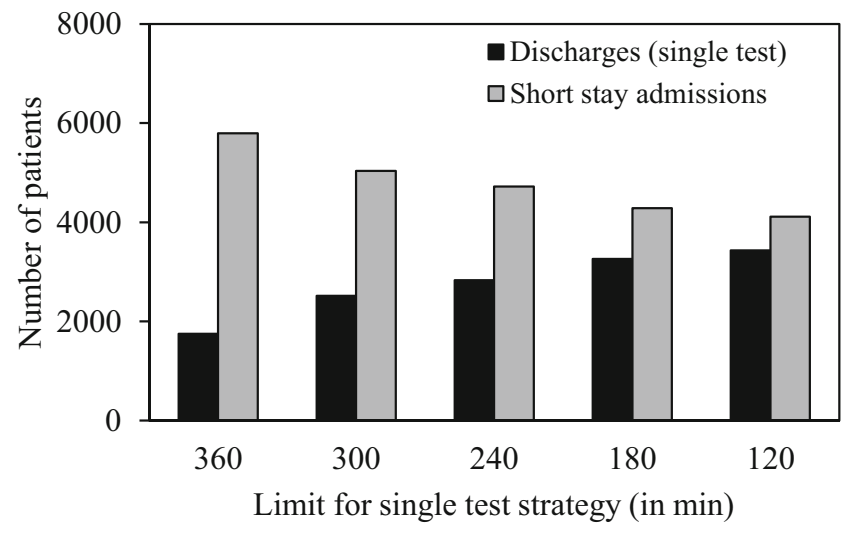

Fig. 13 Changes in the number of short stay admissions and discharges when lowering the time cut-off for single test strategy 


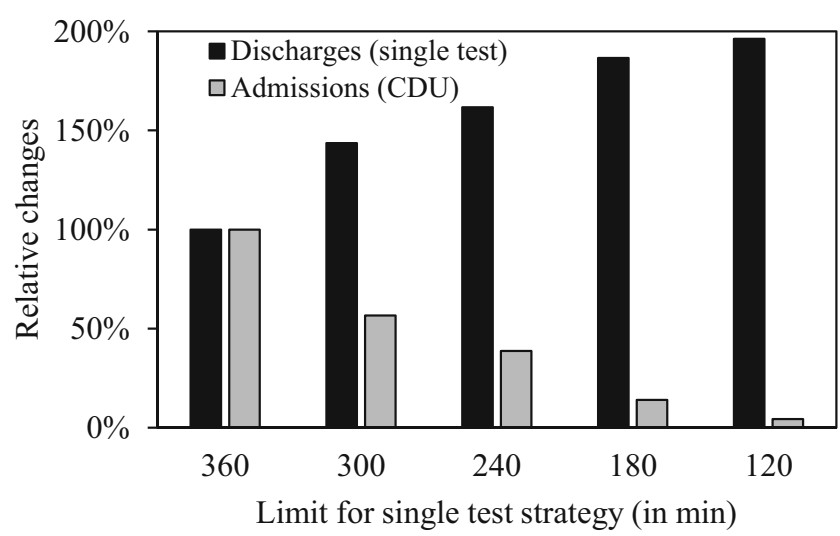

Fig. 14 Relative changes of admissions to CDU and discharges based on a single test - the current setting marks the $100 \%$ level

patients presenting at ED is shorter. Similarly, if the prehospital delay was, on average, shorter, this would imply a higher probability of patients having a blood sample taken later than the acceptable cut-off time and thus potentially more patients to be eligible for a single test rule-out strategy. The outlined impact on admissions and discharges would be less pronounced when there was a longer delay until presentation at ED and subsequently a blood sample drawn and analysed later. Most importantly, however, it is a hospital's decision whether or not to be willing to decide on the basis of a single troponin result given a time delay between onset of pain and the first blood sample taken. This must not be interpreted as a recommendation to patients to delay their attendance to ED in order to potentially save time they spent in ED.

\section{Discussion and outlook}

We presented a DES model as part of a bigger study looking at ED pathways for patients presenting with chest pain. A generic DES model was tailored to represent the situation at a specific trust. We performed a number of what-if analyses and investigated the impact of introducing a single test rule-out strategy where the time cut-off (i.e. the acceptable timespan between onset of pain and the first sample at ED) was lowered from 6 hours down to 2 hours. The currently applied time limit of six hours for single test ruleout strategies is historical and evidence is available that the new high-sensitivity troponin tests can reliably rule out AMI at three hours after symptom onset and shorten patient waits. Given that the reduction of the time threshold can be achieved with low additional risk for patients (see for reference the studies mentioned in Section 2.1), already small reductions of the time thresholds for single test rule-outs can significantly increase the number of discharges based on a single (negative) test.
It should be noted, however, that the model does not allow to assess the additional level of risk. Therefore, clinicians have to make the final decision which time cut-off for single test strategies is acceptable - this might also vary across different hospitals. The critical input factor is the time between onset of worst pain and the first blood sample taken at ED. The positive effects of avoided admissions demonstrated in this paper might become more obvious in settings where there are commonly longer pre-hospital delays. Finally, the high-sensitivity troponin test is just one of many factors by which patients presenting with chest pain are managed within ED. Many such patients can be managed without recourse to it. Our conclusions do not amount to a recommendation to delay hospital attendance when experiencing chest pain, rather a central aim of our study is to help trusts expedite high-sensitivity troponin testing for those patients who will benefit.

Quantifying the key drivers to decide whether or not a patient is admitted could be further investigated using dedicated approaches such as Data Mining or Machine Learning. This could help to refine the simulation model with its decision logic in order to explore how additional knowledge about input factors would affect the accuracy of the model output. Together with an analysis of the time since onset of pain and its effects on the outcome, this could underline the positive impact of our study. Finally, the developed DES model can easily be tailored around trust-specific settings which makes it widely applicable, especially for ACS pathways. It serves primarily as an evaluation tool when looking at a possible implementation of pathway changes at some of the collaborating trusts. The findings from our project were reported directly to the SW AHSN and it is hoped that these will inform further policy directives across the studied hospitals and provide a basis for a subsequent implementation phase of the work.

Acknowledgements The authors would like to acknowledge the engagement from members of staff at acute hospitals in the South West of England. In particular, for providing helpful insights into practical work and for making data accessible which helped to develop discrete event simulation models and to carry out this research. This publication is based on a project funded by the South West Academic Health Science Network (SW AHSN). The work of the authors is also funded by the National Institute for Health (NIHR) Collaboration for Leadership in Applied Health Research and Care South West Peninsula at the Royal Devon and Exeter NHS Foundation Trust. The views expressed are those of the authors and not necessarily those of the NHS, the NIHR, the SW AHSN or the Department of Health.

Open Access This article is distributed under the terms of the Creative Commons Attribution 4.0 International License (http:// creativecommons.org/licenses/by/4.0/), which permits unrestricted use, distribution, and reproduction in any medium, provided you give appropriate credit to the original author(s) and the source, provide a link to the Creative Commons license, and indicate if changes were made. 


\section{Appendix}

We modelled the change of troponin over time for patients not having either STEMI (ST segment elevation myocardial infarction) or NSTEMI (non-ST segment elevation myocardial infarction) using moving average processes $(M A[q])$. [24] recommends a cut-off level of 14ng/litre for troponin. Also, studies report that this is a natural and non-elevated concentration in the blood and can therefore be used as a cut-off level [37]. The normal level of troponin is denoted as $\theta$ and the error terms $\epsilon$ have a mean of $\mu=0$ and a standard deviation of $\sigma=1$. The following equation describes the variation of troponin over time in terms of $X_{t}$ :

$$
\left(X_{t}\right)=\theta+\epsilon_{t}-\alpha \epsilon_{t-1}-\beta \epsilon_{t-2}-\gamma \epsilon_{t-3}-v_{t-4} \epsilon_{t-4}
$$

Figure 15 exemplifies different moving average processes with a mean of $\theta=14$. As per the model, we distinguish patients with cardiac and non-cardiac causes of chest pain. The latter group can be further divided in musculoskeletal and other origins. In terms of weights $\alpha$ through $\gamma$ decreasing effects of previous error terms can be modelled. The baseline level of troponin, however, does depend on additional factors such as age or co-morbidities which can easily be integrated into the model in terms of distinct troponin profiles. Diagnostic experts of our team suggested that age-dependent troponin profiles should be included with further studies, but at the same time confirmed that the variation shown in Fig. 15 accurately captures variation if age categories of patients are omitted.

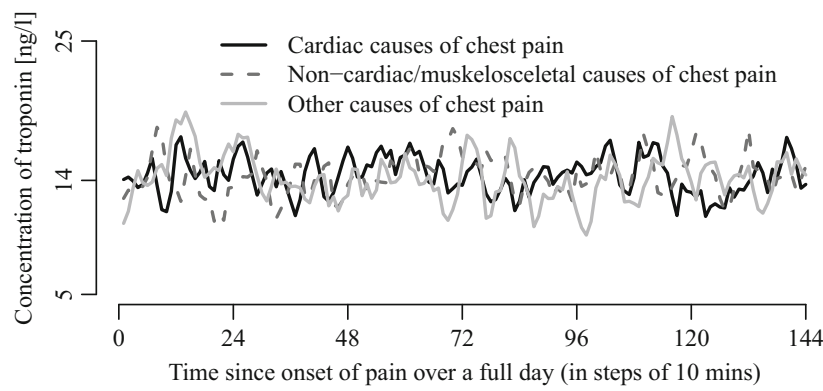

Fig. 15 Natural variation of troponin with low-risk patients modelled in terms of Moving Average processes. The different lines represent independent simulations for different patient

\section{References}

1. Abo-Hamad W, Arisha A (2013) Simulation-based framework to improve patient experience in an emergency department. Eur $\mathbf{J}$ Oper Res 224(1):154-166

2. Academic Health Science Network South West (2016) Study investigates the effective diagnosis of heart attacks in patients attending emergency departments. web link: http://wwwswahsn $\mathrm{com} /$ study-investigates-the-effective-diagnosis-of-heart-attacks-inpatients-attending-emergency-departments/, last visit 28/06/2016
3. Al-Refaie A, Fouad RH, Li MH, Shurrab M (2014) Applying simulation and dea to improve performance of emergency department in a Jordanian hospital. Simul Model Pract Theory 41:59-72

4. Ashour OM, Kremer GEO (2013) A simulation analysis of the impact of fahp-maut triage algorithm on the emergency department performance measures. Expert Syst Appl 40(1):177-187

5. Best AM, Dixon CA, Kelton WD, Lindsell CJ, Ward MJ (2014) Using discrete event computer simulation to improve patient flow in a Ghanaian acute care hospital. Am J Emerg Med 32(8):917922

6. Bhattacharjee P, Ray PK (2014) Patient flow modelling and performance analysis of healthcare delivery processes in hospitals: a review and reflections. Comput Ind Eng 78:299-312

7. Blasak RE, Armel WS, Starks DW, Hayduk MC (2003) The use of simulation to evaluate hospital operations between the emergency department and a medical telemetry unit. In: Proceedings of the 2003 Winter Simulation Conference Vols 1 and 2. Ieee, New York

8. Body R, Carley S, McDowell G, Pemberton P, Burrows G, Cook G, Lewis PS, Smith A, Mackway-Jones K (2014) The manchester acute coronary syndromes (macs) decision rule for suspected cardiac chest pain: derivation and external validation. Heart 100(18):1462-8

9. Brenner S, Zeng Z, Liu Y, Wang J, Li J, Howard PK (2010) Modeling and analysis of the emergency department at university of kentucky chandler hospital using simulations. J Emerg Nurs 36(4):303-310

10. Cardinaels EP, Mingels AM, van Rooij T, Collinson PO, Prinzen FW, van Dieijen-Visser MP (2013) Time-dependent degradation pattern of cardiac troponin $\mathrm{t}$ following myocardial infarction. Clin Chem 59(7):1083-90

11. Chenevier-Gobeaux C, Meune C, Lefevre G, Doumenc B, Sorbets E, Peschanski N, Ray P (2016) A single value of high-sensitive troponin $\mathrm{t}$ below the limit of detection is not enough for ruling out non st elevation myocardial infarction in the emergency department. Clinical Biochemistry (in press)

12. Deflitch SAP, Reddy MC, J C (2010) A systematic review of simulation studies investigating emergency department overcrowding. Simulation 86(8-9):559-571

13. Degel D, Wiesche L, Rachuba S, Werners B (2015) Timedependent ambulance allocation considering data-driven empirically required coverage. Health Care Manag Sci 18(3):444-458

14. Department of Health (2004) Improving emergency care in england. House of commons, committee of public accounts, Sixteenth Report of Session 2004-05, published on 30 March 2005

15. Fletcher A, Worthington D (2009) What is a generic hospital model? - a comparison of generic and specific hospital models of emergency patient flows. Health Care Manag Sci 12(4):374391

16. Goodacre S, Cross E, Arnold J, Angelini K, Capewell S, Nicholl J (2005) The health care burden of acute chest pain. Heart 91(2):229-230

17. Gul M, Guneri AF (2015) A comprehensive review of emergency department simulation applications for normal and disaster conditions. Comput Ind Eng 83:327-344

18. Hitchcock T, Rossouw F, McCoubrie D, Meek S (2003) Observational study of prehospital delays in patients with chest pain. Emerg Med J 20(3):270-273

19. Hong CC, Sultana P, Wong AS, Chan KP, Pek PP, Ong ME (2011) Prehospital delay in patients presenting with acute st-elevation myocardial infarction. Eur J Emerg Med 18(5):268-71

20. Komenda I, Knight V, Williams HM (2015) Compliance with national guidelines for stroke in radiology. Operations Research for Health Care 6:33-39

21. McKinley S, Aitken LM, Marshall AP, Buckley T, Baker H, Davidson PM, Dracup K (2011) Delays in presentation with 
acute coronary syndrome in people with coronary artery disease in Australia and New Zealand. Emerg Med Australas 23(2): 153-61

22. Mustafee N, Katsaliaki K (2011) Applications of simulation within the healthcare context. J Oper Res Soc 62(8):1431-1451

23. NICE (2010) Chest pain of recent onset: assessment and diagnosis. NICE clinical guideline, pp 1-46

24. NICE (2014) Myocardial infarction (acute): Early rule out using high-sensitivity troponin tests (elecsys troponin $t$ high-sensitive, architect stat high sensitive troponin-i and accutni+3 assays). NICE diagnostics guidance 15:1-53

25. Norouzzadeh S, Garber J, Longacre M, Akbar S, Riebling N, Clark R (2014) A modular simulation study to improve patient flow to inpatient units in the emergency department. J Hosp Admit Manage 3(6):205-215

26. Olivieri F, Galeazzi R, Giavarina D, Testa R, Abbatecola AM, Çeka A, Tamburrini P, Busco F, Lazzarini R, Monti D, Franceschi C, Procopio AD, Antonicelli R (2012) Aged-related increase of high sensitive troponin $t$ and its implication in acute myocardial infarction diagnosis of elderly patients. Mech Ageing Dev 133(5):300-305

27. Perkins-Porras L, Whitehead DL, Strike PC, Steptoe A (2009) Prehospital delay in patients with acute coronary syndrome: Factors associated with patient decision time and home-to-hospital delay. Eur J Cardiovasc Nurs 8(1):26-33

28. Rachuba S, Salmon A, Zhelev Z, Pitt M (2016) Simulating single test rule-out strategies for chest pain patients at Emergency Departments. In: Proceedings of the 8th Simulation Workshop (SW16), pp 135-148

29. Rashwan W, Abo-Hamad W, Arisha A (2015) A system dynamics view of the acute bed blockage problem in the irish healthcare system. Eur J Oper Res 247(1):276-293

30. Saghafian S, Austin G, Traub SJ (2015) Operations research/management contributions to emergency department patient flow optimization: Review and research prospects. IIE Transactions on Healthcare Systems Engineering 5(2):101-123

31. Shah ASV, Anand A, Sandoval Y, Lee KK, Smith SW, Adamson PD, Chapman AR, Langdon T, Sandeman D, Vaswani A, Strachan FE, Ferry A, Stirzaker AG, Reid A, Gray AJ, Collinson PO, McAllister DA, Apple FS, Newby DE, Mills NL (2015) Highsensitivity cardiac troponin $\mathrm{i}$ at presentation in patients with suspected acute coronary syndrome: a cohort study. The Lancet 386(10012):2481-2488

32. Sorup CM, Sepúlveda Estay DA, Jacobsen P, Anderson PD (2015) Balancing patient flow and returning patients: A system dynamics study on emergency department crowding factors. Health Care Management Science

33. Westwood M, van Asselt T, Ramaekers B, Whiting P, Thokala P, Joore M, Armstrong N, Ross J, Severens J, Kleijnen J (2015) High-sensitivity troponin assays for the early rule-out or diagnosis of acute myocardial infarction in people with acute chest pain: a systematic review and cost-effectiveness analysis. Health Technol Assess 19(44):1-234

34. Wiler JL, Griffey RT, Olsen T (2011) Review of modeling approaches for emergency department patient flow and crowding research. Acad Emerg Med 18(12):1371-9

35. Xu RY, Zhu XF, Yang Y, Ye P (2013) High-sensitive cardiac troponin t. J Geriatr Cardiol : JGC 10(1):102-109

36. Yang KK, Lam SSW, Low JMW, Ong MEH (2016) Managing emergency department crowding through improved triaging and resource allocation. Operations Research for Health Care 10:1322

37. Zhelev Z, Hyde C, Youngman E, Rogers M, Fleming S, Slade T, Coelho H, Jones-Hughes T, Nikolaou V (2015) Diagnostic accuracy of single baseline measurement of elecsys troponin $t$ high-sensitive assay for diagnosis of acute myocardial infarction in emergency department: systematic review and meta-analysis. BMJ 350 